\title{
Role of high dose corticosteroids and visual outcome in cases of traumatic optic neuropathy with delayed presentation in a tertiary eye care centre
}

\author{
Kubrey S.S. ${ }^{1}$, Ahuja B. ${ }^{2}$, Singh D. ${ }^{3}$ \\ ${ }^{1}$ Dr. S.S. Kubrey, Associate Professor, Department of Ophthalmology, Gandhi Medical College, Bhopal (M.P.), \\ ${ }^{2}$ Dr. Bharti Ahuja, Senior Resident, Department of Ophthalmology, Chhindwara Institute of Medical Sciences, \\ Chhindwara (M.P.), ${ }^{3}$ Dr. Dheerendra Singh, Junior Resident, Department of Ophthalmology, Gandhi Medical College, \\ Bhopal (M.P.)
}

Corresponding Author: Dr. Bharti Ahuja, Senior Resident Department of Ophthalmology Chhindwara Institute of Medical Sciences, Chhindwara (M.P.) Email: bhartiahuja2@gmail.com

\begin{abstract}
Background: Optic nerve injuries occur in the setting of head injury which is often a consequence of road traffic accidents or falls. Traumatic optic neuropathy (TON) is a potentially blinding complication of head and orbital trauma. Different treatment approaches like different dosages of steroids, surgical decompression and observation alone have been suggested but there is no standardized protocol till date. Object: To study the clinical profile, risk factors, visual outcome in a series of forty five patients of Traumatic Optic Neuropathy (TON) after medical management at our tertiary centre. Methods: Forty five patients reported to outpatient department from January 2018 to June 2019 who have a history of trauma were studied prospectively. After relevant and detailed ocular examination, Intravenous methyl prednisolone 1 gram for 5 days and thenoral prednisolone $1 \mathrm{mg} / \mathrm{kg}$ in tapering doses for two weeks was administered in all patients irrespective of duration of presentation to the hospital after trauma. Results: The age group involved was predominantly 16 to 45 years $(80 \%)$ with a mean age of 35.31 years. Range of follow up was seen from 5 days to 5 months. Males (91\%) were outnumbered. Road traffic accidents $(73.33 \%)$ were found to be the most common cause for TON in our series. Of 45, 10 patients were lost to follow up, rest 35 patients who have been followed steroid regime, 18 (51.43\%) showed visual improvement (12 patients had $>/=2$ line improvement, rest 06 patient had one line improvement). $17(48.57 \%$ ) patients did not show any improvement. 08 patients were same (no perception of light) till the final follow up. Conclusion: IV methyl prednisolone along with oral steroids as proposed by ONTT has been found effective in our case series of TON even in patients who have very low initial visualacuity and who presented late even after 24 hours of injury till the irreversible damage to optic nerve.No major side effects have been encountered with this steroid regimen. High dose steroid has proven its role in traumatic optic neuropathy.
\end{abstract}

Keywords: Methyl prednisolone, Optic neuropathy, Road traffic accidents, Trauma, Visual outcome

\section{Introduction}

Traumatic Optic Neuropathy (TON) following craniofacial injuries was first described by "Hippocrates". Traumatic optic neuropathy refers to an acute injury to the optic nerve secondary to trauma (blunt or penetrating) which occurs without external or initial ophthalmoscopic evidence of injury to the eye or its nerve. It is an uncommon but devastating cause of permanent visual loss following contusive injuries to the head, particularly forehead caused by impact to the

Manuscript received: $18^{\text {th }}$ June 2019

Reviewed: $28^{\text {th }}$ June 2019

Author Corrected: $4^{\text {th }}$ July 2019

Accepted for Publication: $8^{\text {th }}$ July 2019 optic nerve. The impact transmits a shockwave to the optic canal damaging the optic nerve. Injury may be direct or indirect and visual loss may be partial or complete. It can be classified depending on the site of injury (i.e. optic nerve head, intraorbital, intracanalicular or intracranial) or according to the mode of injury (i.e. direct or indirect) $[1,2]$. The most common form of traumatic optic neuropathy is indirect damage to the optic nerve and has been reported following $0.5 \%$ to $5 \%$ of all closed head traumas [3, 4]. Indirect injury results from transmission of forces to the nerve from a distant site without disruption of normal tissue 
structures. Clinical features of TON include visual loss, dyschromatopsia, relative afferent pupillary defect (except in the case of a bilateral TON), visual field defects. The optimal treatment of traumatic optic neuropathy remainscontroversial. There has been no conclusive evidence for standardized treatment protocol due to lacking of large randomised controlled trials of management as a result of low incidence of this condition [5]. Observation, corticosteroids, treatment and decompression of the optic nerve have been advocated and significant recovery of vision has been found in those treated with corticosteroids, optic nerve decompression, or both as compared to observation alone [6]. The present study attempted to report a series of forty five patients of TON who received treatment in the form of corticosteroids at our tertiary eye care centre to study the clinical profile ofdisease, timing of presentation and to assess the final visual outcome.

\section{Materials and Methods}

Study design: A prospective hospital based observational study.

Ethical Approval was granted by the Institutional Review Board.

Duration of study: January 2018 to June 2019

Inclusion criteria: A total of forty five patients reporting to the Outpatient department of Ophthalmology in Hamidia hospital Bhopal with or without head injury, already diagnosed cases of traumatic optic neuropathy from elsewhere and those who can be treated medically were enrolled.

\section{Results}

Table-1: Demographics \& baseline visual acuity.

\begin{tabular}{|l|c|}
\hline & Frequency (\%) $\mathbf{n = 4 5}$ \\
\hline Mean age (years) & $\mathbf{3 5 . 3 1}$ \\
\hline Sex Fale & $41(91)$ \\
\hline \multicolumn{1}{|c|}{ Female } & $4(8.9)$ \\
\hline Eyeft & $23(51.11)$ \\
\hline \multicolumn{1}{|c|}{ Fall } & $22(48.9)$ \\
\hline Injury type $\quad$ RTA & $33(73.33)$ \\
\hline \multicolumn{1}{|c|}{ Assault } & $07(15.6)$ \\
\hline Fire Cracker Injury & 04 \\
\hline Baseline Visual Acuity (VA) $(\mathrm{n}=35) 6 / 12$ to $6 / 18$ & 01 \\
\hline $6 / 24$ to $6 / 36 \quad 07$ \\
\hline $6 / 60$ & 02 \\
\hline$<6 / 60$ & 02 \\
\hline No PL & $25(55.56)$ \\
\hline
\end{tabular}

Exclusion criteria: Patients who did not give consent for either admission or treatment and those who have preexisting ocular abnormalities that affect visual function.

Salient demographic data (detailed clinical history of injury with timing of presentation to clinic ),including Visual acuity assessment,torch light examination including RAPD (relative afferent pupillary Defect) assessment, extraocular motility, slit lamp examination, colour vision testing, fundus examination, $\mathrm{b}$ scan ultrasonography, Automated perimetry (30-2), VEP and CT/MRI wherever required/ possible was done accordingly. Visual acuity was the main outcome measure of the study, which was measured by Snellen chart on admission, immediately after treatment and 4 weeks later.

A written informed consent was taken prior to starting of medical treatment. Intravenous Methylprednisolone 1 gram was given for 5 days (diluted in $100 \mathrm{ml}$ Normal saline over 45 minutes).

Pulse and Blood Pressure recorded prior to infusion and monitored. Then oral prednisolone $1 \mathrm{mg} / \mathrm{kg}$ in tapering doses for two weeks was administered.Patients were examined every day during hospitalization and then followed every week till the end of study period.

Statistical analysis: Data were presented as percentage (\%) for qualitative variables, mean and standard deviation for continuous variables and Chi-square test for frequencies. $p$ value $<0.05$ was considered statistically significant. 
Data of 45 patients were analysed. The age group involved was predominantly 16 to 45 years $(80 \%)$ with a mean age of 35.31 years. Range of follow-up was seen from 5 days to 5 months. Of 45 , all patients had an indirect optic nerve injury resulted from blunt trauma to head, one had an open globe injury also. Forty one $(91 \%)$ were male and four were female.

Range of timing of initial presentation to the hospital for the initiation of treatment was seen from 03 hours to 05 months. $31 \%$ patients presented within less than 24 hours and $48.9 \%$ afterwards (Table- 1 )

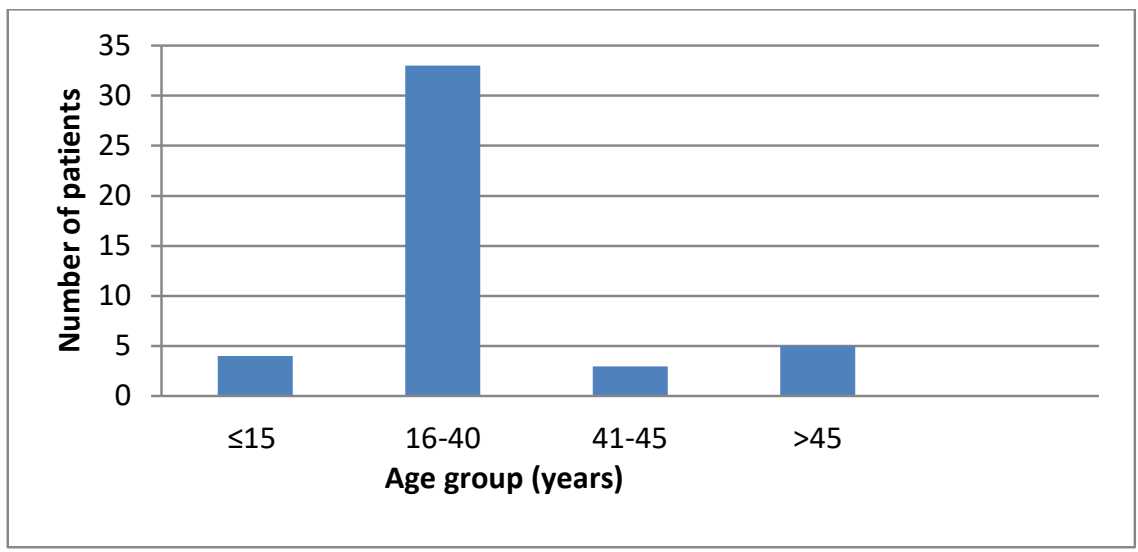

Figure-1: Age group distribution

Ten patients lost to follow up after initial presentation. The major causes of trauma were Road traffic accidents (RTA) (73.33\%) followed by falls (15.56\%). Majority of patients were labourer (44.4\%) by occupation. Out of 45 patients, 25 $(55.56 \%)$ had visual acuity $<6 / 60$ (excluding No PL) at presentation. RAPD was seen in 37 patients and APD in 08 patients who had no perception of light at presentation.

Visual fields and colour vision testing attempted in all patients at presentation, results are not very reliable due to low vision. Extraocular motility tested in all patients, found normal in all 43, restriction seen in only 2 cases.Visual evoked potential (VEP) testing shown decreased amplitude, increased latency in 14.cases and was not possible in 20cases due to decreased vision, and normal in 01 patient.

Best corrected visual acuity at final follow up was measured as primary outcome. Regime of three doses of Intravenous methylprednisolone (high dose) with oral steroidshas been given to 35 patients.

Out of which $18(51.43 \%)$ showed visual improvement (12 patients had $\geq 2$ line improvement, rest 06 patient had one line improvement) (Table 2). 17 (48.57\%) patients did not show any improvement. 08 patients were same (no perception of light till the final follow up).

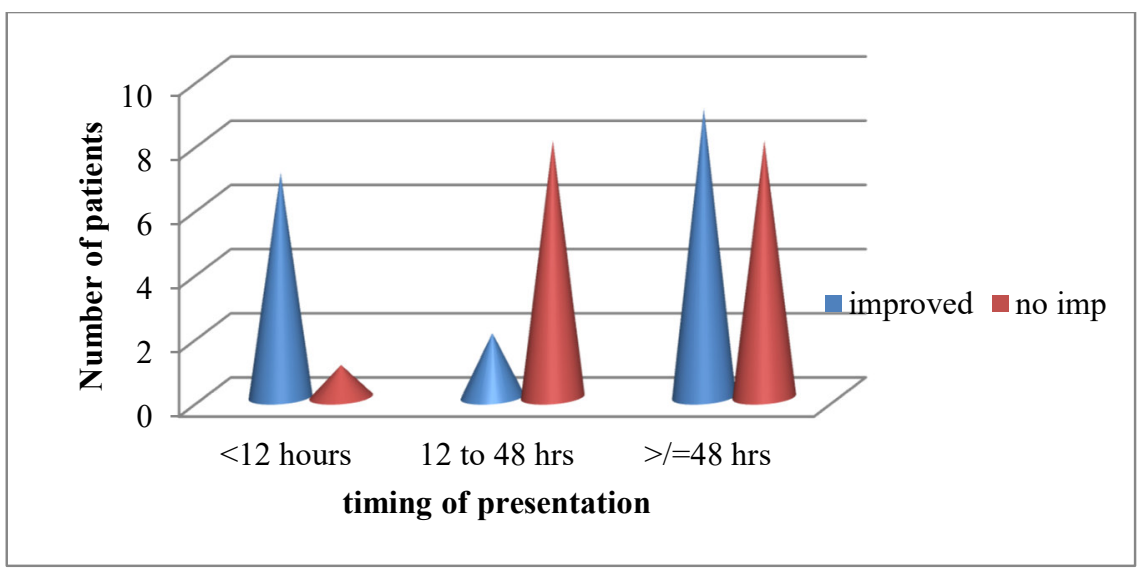

Figure-2: Improvement in visual outcome versus timing of presentation 
Original Research Article

Table-2: Significant final visual outcome after steroid therapy $(\mathrm{N}=12)$

\begin{tabular}{|c|c|c|c|}
\hline S.NO. & $\begin{array}{c}\text { Visual acuity(VA) at } \\
\text { presentation }\end{array}$ & $\begin{array}{c}\text { Timing of presentation to } \\
\text { hospital after injury (hours) }\end{array}$ & VA Post steroid therapy \\
\hline 1 & No PL & $>96$ & FC3 ft $(1.9)$ \\
\hline 2 & PL(2.8) & 5 & $6 / 12(0.3)$ \\
\hline 3 & PL(2.8) & 5 & $6 / 36(0.80)$ \\
\hline 4 & PL(2.8) & 5 & $6 / 9(0.2)$ \\
\hline 5 & PL(2.8) & 24 & $6 / 24(0.60)$ \\
\hline 6 & HM(2.3) & 96 & FC 3 ft $(1.9)$ \\
\hline 7 & FC FC & $>96$ & $1 / 60(1.8)$ \\
\hline 8 & FC $2 \mathrm{ft}(2.0)$ & 12 & $3 / 60(1.30)$ \\
\hline 9 & FC 3 ft (1.9) & 5 & $5 / 60(1.1)$ \\
\hline 10 & $4 / 60(1.2)$ & $>96$ & $6 / 24(0.60)$ \\
\hline 11 & $6 / 18(0.5)$ & 6 & $6 / 9(0.2)$ \\
\hline 12 & $6 / 12(0.3)$ & $6 / 6(0.0)$ \\
\hline
\end{tabular}

NO PL $=$ No Perception of light

$\mathrm{FC} \mathrm{FC}=$ finger count close to face

$\mathrm{PL}=$ perception of light

$\mathrm{HM}=$ Hand movements

$\mathrm{FC}=$ Finger Count

\section{Discussion}

TON is considered an ophthalmic emergency, often seen in the setting of head trauma and no standardized treatment protocol has been mentioned in the literature for this entity. Optimal management of TON is still the subject of debate. Despite these persisting uncertainties, the main treatment options in current use for TON are as follows: (1) systemic steroids of varying doses, duration, and mode of administration; (2) surgical decompression of the optic canal; (3) a combination of steroids and surgery; and (4) observation alone (i.e., conservative management).

Here authors have attempted to see the effect of steroids in cases of TON prospectively to our tertiary centre even in those cases who presented late (even after 48 hours).

The vast majority of affected patients are young males $(79 \%$ to $85 \%)$ in theirearly thirties in a national epidemiological survey of TON in the UK. The most common causes of TON in adults are motor vehicle and bicycle accidents (49\%), falls (27\%) and assaults $(13 \%)[7,8] .73 .3 \%$ cases were found to be due to road traffic accidents in present study. Similar findings were showed in Sadeghi-Tari study [2,9,10] and $80 \%$ of our patients were between 16 to 45 years, males (91\%), which is found to be seen consistent with the previous studies $[7,11]$.
Steroids have been used both on its own and in combination with surgical optic nerve decompression either pre-, intra-, or postoperatively $[16,21]$. Steroid regimens can be classified as (based on the initial daily dose of methyl prednisolone used):

1. low dose $(<100 \mathrm{mg})$

2. Moderate(100-499mg)

3. High dose(500-1999mg)

4. Very high dose(2000-5399mg)

5. Mega dose (>5400mg)

National Acute Spinal Cord Injury Study (NASCS) [12, $13,14]$ concluded that megadose steroids within 8 hours of injury showed better neurological outcomes. CORTICOSTEROID RANDOMISATIONAFTER SIGNIFICANT HEAD INJURY STUDY (CRASH) $[15,16]$ - A large randomized placebo controlled study again evaluating megadose of steroids in traumatic brain injury, had been stopped due to increased mortality in patients received megadose.

In the present study, high dose [17] regimen similar to ONTT (OPTIC NEURITIS TREATMENT TRIAL) trial has followed (1 gm of IV methyl prednisolone for 3-5 days followed by $1 \mathrm{mg} / \mathrm{kg}$ oral prednisolone in tapering dose over 2 weeks). 
The International Optic Nerve Trauma Study (IONTS) is the largest, prospective, multicenter study of TON published to date [8]. It was intended to be a randomized controlled trial, but it had to be converted to an observational study after 2 years owing to recruitment failure. The analysis included a total of 133 people with indirect TON treated within 7 days of injury and categorized into three groups: untreated $(n=9)$, steroids $(n=85)$, or optic canal decompression surgery $(n=33)$. The majority of patients in the steroid group had either a megadose $(40 \%)$ or very high-dose regimen $(18 \%)$, and all the participants in the surgical group, except for one, also received steroids. Follow-up data were available for 104 cases at 1 month and for 40 cases at 6 months. After adjustment for baseline visual acuity, no significant differences were found between the three treatment groups. A three-line increase in visual acuity or more occurred in $57 \%$ of the untreated group, $52 \%$ of the steroid group, and $32 \%$ of the surgery group [8]. Some case series have reported higher improvement rates with steroids, most published figures (44-62\%) are comparable with IONTS [7, 9, 18, 19, $20]$.

The criteria for visualimprovement following treatment was not clearlydefined in the majority of published data. However fewstudies showed improvement as an increase in 1 to 3 lines in visual acuity $[2,8]$. Methylprednisolone is preferred as the initial treatment of choice because of its neuroprotective mechanism [22]. But the exact mechanism of its neuroprotective action is not clear. However, itmainly relieves the compression of the optic nerve fiber by reducing the intraneural and extraneural edema. Theother mechanism of its neuroprotective action includes inhibition of the free radicals formation, limitation ofcontusion necrosis of the nerve and blockage ofneuronal death by decreasing the vasospasm [5].

In this case series of 45 patients, 10 were untreated and 35 received corticosteroids. Statistically significant visual improvement was seen in $51.4 \%$ of treated cases.Similar results were seen in a study by Chou etal [9] who concluded that patients in treated group (57\%) did statistically better than untreated patients. The predictors of prognosis seen in this study were presenting visual acuity, timing of presentation to the hospital for initiation of treatment, associated other ocular co-morbidities .However, in the present study also suffered few limitations due to small sample size, wide variation in age group of patients for which response of steroids could have been different.Our results would have been different if the ten patients who lost for follow upalso could have been included.

\section{Conclusion}

IV methyl prednisolone along with oral steroids as proposed by ONTT has been found effective in our case series of TON even in patients who have very low initial visualacuity and who presented late even after 24 hours of injury till the irreversible damage to optic nerve. No major side effects have been encountered with this steroid regime. In the present study concludes that above mentioned treatment shouldalways be considered in diagnosed patients of traumatic optic neuropathy irrespective of the timing of presentation. However, any contraindication to steroid obviously precludes the use. It is a well known fact that high dose of steroids when given early i.e. within $72 \mathrm{hrs}$ of trauma, good visual outcome is noted. In the present study, however, it was observed that in cases of TON even beyond $72 \mathrm{hrs}$ of trauma if steroids are given it can significantly alter the visual prognosis.

\section{Author's contribution}

Dr. S.S. Kubrey: Collaborated on the concept, data collection, data analysis and manuscript preparation.

Dr. Bharti Ahuja: Conceptualization, data analysis and manuscript preparation

Dr. Dheerendra Singh: Collaborated on the concept, data collection, data analysis and manuscript preparation.

\section{Acknowledgement: None}

Funding: Nil, Conflict of interest: Nil Permission from IRB: Yes

\section{References}

1. Sarkies N. Traumatic optic neuropathy.Eye (Lond). 2004;18(11):1122-5. DOI:10.1038/sj.eye.6701571

2. Steinsapir KD, Goldberg RA. Traumatic optic neuropathy.Surv Ophthalmol. 1994; 38(6):487-518. DOI: https: // doi.org/ 10.1016/ 0039-6257(94)90145-7

3. Carta A, Ferrigno L, Salvo M, Bianchi-Marzoli S, Boschi A, Carta F. Visual prognosis after indirect traumatic optic neuropathy. J Neurol Neurosurg Psychiatry. 2003;74(2): 246-8.DOI:10.1136/jnnp. 74. 2.246

4. Kovacić M, Gracner T, Gracner B. Indirect traumatic optic neuropathy--two case report. Coll Antropol. 2001;25 Suppl:57-61.

5. Sadeghi-Tari A, Lashay AR, Tabassi A. Visual outcome of traumatic opticneuropathy in patients treated with intravenous megadose of steroids.Acta Medica Iranica 2005;43(2):110-114. 


\section{Original Research Article}

6. Li KK, Teknos TN, Lai A, Lauretano AM, Joseph MP. Traumatic optic neuropathy: result in 45 consecutive surgically treated patients. Otolaryngol Head Neck Surg. 1999;120(1):5-11.DOI:10.1016 /S0194 -5998(99)70362-1

7. Lee V, Ford RL, Xing W, Bunce C, Foot B. Surveillance of traumatic optic neuropathy in the UK. Eye (Lond). 2010;24(2):240-50. DOI: 10.1038/eye. 2009. 79

8. Levin LA, Beck RW, Joseph MP, Seiff S, Kraker R. The treatment of traumatic optic neuropathy: the International Optic Nerve Trauma Study. Ophthalmology. 1999;106(7):1268-77. DOI: https://doi. org/10. 1016/S0161-6420(99)00707-1

9. Chou PI, Sadun AA, Chen YC, Su WY, Lin SZ, Lee CC. Clinical experiences in the management of traumatic optic neuropathy. Neuro-Ophthalmology. 1996;16(6):325-336.DOI:https://doi.org/10.3109/01 658109609044636

10. Davidson M. The indirect traumatic optic atrophies. Am J Ophthalmol;1938;21(1):7

11. Pirouzmand F. Epidemiological trends of traumatic optic nerve injuries in the largest Canadian adult trauma center. J Craniofac Surg. 2012;23(2):516-20. DOI: 10. 1097/SCS.0b013e31824cd4a7.

12. Bracken MB, Shepard MJ, Collins WF, Holford TR, Young W, Baskin DS, et al. A randomized, controlled trial of methylprednisolone or naloxone in the treatment of acute spinal-cord injury.Results of the Second National Acute Spinal Cord Injury Study.N Engl J Med. 1990; 322 (20):1405-11.DOI:10.1056/ NEJM 199005173222001

13. Bracken MB, Shepard MJ, Holford TR, LeoSummers L, Aldrich EF, Fazl M, et al. Administration of methylprednisolone for 24 or 48 hours or tirilazadmesylate for 48 hours in the treatment of acute spinal cord injury. Results of the Third National Acute Spinal Cord Injury Randomized Controlled Trial. National Acute Spinal Cord Injury Study. JAMA. 1997;277(20):1597-604.
14. Spoor TC, Hartel WC, Lensink DB, Wilkinson MJ. Treatment of traumatic optic neuropathy with corticosteroids. Am J Ophthalmol. 1990;110(6): 665-9. DOI: 10. 1016/s0002-9394(14)77065-5

15. Edwards $\mathrm{P}$, Arango M, Balica L, Cottingham R, ElSayed H, Farrell B, et al. Final results of MRC CRASH, a randomised placebo-controlled trial of intravenous corticosteroid in adults with head injury-outcomes at 6 months. Lancet. 2005;365(9475):1957-9. DOI:10.1016/ S0140-6736(05)66552-X

16. Yu-Wai-Man P, Griffiths PG. Steroids for traumatic optic neuropathy. Cochrane Database Syst Rev. 2011; 19 (1):CD006032. DOI: 10.1002/14651858. CD006032. pub3.

17. Volpe NJ, Levin LA. How should patients with indirect traumatic optic neuropathy be treated? J Neuroophthalmol. 2011;31(2):169-74. DOI: 10.1097/ WNO. 0b013e31821c9b11.

18. Wang BH, Robertson BC, Girotto JA, Liem A, Miller NR, Iliff N, et al. Traumatic optic neuropathy: a review of 61 patients. Plast Reconstr Surg. 2001;107 (7):1655-64.DOI:10.1097/00006534-200106000-00003

19. Cook MW, Levin LA, Joseph MP, Pinczower EF. Traumatic optic neuropathy.A meta-analysis.Arch Otolaryngol Head Neck Surg. 1996;122(4):389-92. DOI: 10.1001/archotol.1996.01890160031006

20. Spoor TC, Hartel WC, Lensink DB, Wilkinson MJ. Treatment of traumatic optic neuropathy with corticosteroids. Am J Ophthalmol. 1990;110(6):665-9.DOI: 10.1016/s0002-9394(14)77065-5

21.Yu-Wai-Man P, Griffiths PG. Surgery for traumatic optic neuropathy.CochraneDatabaseSyst Rev. 2005;19 (4):CD005024.DOI:10.1002/14651858.CD005024.pub2

22. Hall ED. The neuroprotective pharmacology of methylprednisolone.J Neurosurg. 1992;76(1):13-22. DOI: 10.3171/jns.1992.76.1.0013

\section{How to cite this article?}

Kubrey S.S, Ahuja B, Singh D.Role of high dose corticosteroids and visual outcome in cases of traumatic optic neuropathy with delayed presentation in a tertiary eye care centre. Trop J Ophthalmol Otolaryngol.2019;4(3):190195.doi:10.17511/jooo.2019.i03.02 